\title{
L'agriculture familiale et l'agriculture durable en Amérique du Nord: vers une agriculture "environnementalement correcte"
}

\section{Family farming and sustainable farming in North America: toward a "environmentally-correct" agriculture}

\author{
Bruno JEAN*
}

\begin{abstract}
RESUME
L'auteur analyse les mesures mises en oeuvre aux États-Unis et au Canada et qui vont dans le sens de l'auto-responsabilisation des agriculteurs face à la protection de l'environnement et des ressources agricoles renouvelables. L'auteur défend la thèse que l'émergence de cette responsabilité environnementale des agriculteurs nord-américains peut donner naissance à une agriculture durable ou "environnementalement correcte" qui se fera dans le cadre du système agoalimentaire actuel et qui repose des fermes familiales modernisées. Après avoir décrit les principales mesures du dispositif agroenvironnemental du Farm Bill américain qui met l'accent sur la capacité de "stewarship" des agriculteurs (gestion avisée des terres au sens de "ménagement" comme le disaient Olivier de Serres, un fondateur de l'agronomie moderne) et dont les mesures canadiennes se font aussi l'écho, l'auteur conclue en montrant que l'agriculture familiale est une forme sociale de production agricole proche du modèle théorique de l'agriculture durable.
\end{abstract}

\begin{abstract}
The basic components of the American and Canadian public regulation regarding environment and agricultural ressources protection are review in this paper dealing also with the question of farmer's liability to natural ressources preservation. The author take the position that the rise of environnemtal responsability of farmers can be the base for the development of a truly sustainable agriculture or a "environmentally-correct agriculture". Therefore, this new agriculture model can take place inside the contempory agro-food system based on modern and productive families farms. After a brief description of the regulation under the American Farm Bill introducing the concept of farmer's "stewardship" (a notion not far away to the concept of "good management" or to the old French wording of "ménagement" of land launched by Olivier de Serres, a father of the French modern agfronomy), the article describe the Canadian approach which is quite similar. Finally, the author draw a conclusion in what he stand that the contempory family farming system can be viewed as a social form of agricultural production close to the theorical model of sustainable farming.
\end{abstract}

* Directeur du Doctorat en développement régional. Université du Québec à Rimouki, Québec, Canada. 
En Amérique du Nord, malgré les grands espaces, les terres cultivables ne sont pas si étendues, et leurs rendements sont loin, notamment pour la production céréalière, d'atteindre les niveaux de productivité observés en Europe. Au Canada, les terres agricoles occupent moins de 3\% du territoire national. La politique agricole a souvent consisté en des mesures de protection d'une quantité plutôt limitée des terres arables, allant des dispositifs réglementaires plus ou moins incitatifs au zonage agricole intégral sanctionné par la loi. C'est le cas du Québec avec sa Loi de protection du territoire agricole qui interdit que les terres situées dans la "zone verte" définie par la loi servent à d'autres usages que les activités agricoles. Avant de mettre en place des mesures pour que les terres soustraites aux cultures commerciales intensives ou les terres abandonnées en régions marginales gardent leur potentiel tout en servent à d'autres usages (comme le support de la diversité biologique en permettant la reproduction de la faune et de la flore), il fallait s'assurer que les terres cultivables ne subissent pas une concurrence déloyale des autres activités.

L'approche agri-environnementale nord-américaine pourrait être qualifiée de conservationniste. En effet, ou bien il s'agit de conserver les ressources agricoles d'une région donnée dans leur intégralité, le cas du Québec mentionné ici, ou certaines parties du territoire ayant une grande valeur pour la reproduction des écosystèmes, en les soustrayant aux cultures commerciales intensives. Nous pensons ici au CRP (Conservation Reserve Program) mis en oeuvre dans la politique agricole des États-Unis. Cette approche conservationniste, qui à l'échelle de l'histoire américaine, est longue - les parcs et réserves sont plus que centenaires - semble encore exercer un déterminisme sur le modèle nord-américain de gestion de l'environnement agricole.

Nous nous proposons ici de présenter sommairement quelques-unes de ces mesures mises en oeuvre aux États-Unis et au Canada et qui, nous l'espérons, poseront les éléments propres à étayer l'hypothèse que si une agriculture durable doit généraliser, ce que nous pensons par ailleurs, elle se fera sur la base du système agro-alimentaire actuel qui repose sur des fermes familiales plus aptes à respecter le modèle théorique d'une agriculture durable ou "environnementalement correcte". L'analyse proposée ici, reposant sur une étude réalisée en $1995-96^{1}$, garde toute son actualité car nous avons constaté comment cette idée d'un lien entre l'agriculture familiale et les perspectives qu'elle offrait pour la conversionà une agriculture durable devenaient une hypothèse réaliste au récent congrès mondial de sociologie rurale de Rio de Janario.

Un autre questionnement, et dont les termes ont aussi été posés par Christian Deverre ${ }^{2}$, nous servira aussi de grille de lecture des diverses initiatives nord-américaines dans le domaine de la protection de l'environnement agricole et rural. Il s'agit de la tension entre deux approches foncièrement opposées dans la conception de la politique agri-environnementale, celle répressive de la judiciarisation, où les agriculteurs peuvent faire face à des accusations d'actes criminels, ou celle incitative, où les mêmes agriculteurs sont réputés compétents et capables de d'établir et de faire respecter au sein de leur profession un code des bonnes pratiques "agri-environnementales" et où ils se donnent les moyens d'assumer leurs responsabilités avant de se les faire imposer par la loi.

'Cet article reprend des éléments d'une étude réalisée dans en France lors d'un d'un séjour de recherche à l'LNRA au Département Systèmes Agraires et Développement en 1995-95 et publié dans la revue Études rurales et de notre expérience comme coordonnateur avec Maria de Nazareth Wanderley (Brésil), Babis Kasimis (Grèce) et Susana Aparicio (Chile) du Worshop "The familiy farming in the late modernity" que avons organisé dans le cadre du Congrès mondial de sociologie rurale de Rio de Janerio à l'été 2000. Il reprend aussi des travaux qui ont été, pour une partie, été publié dans notre livre: Territoires d'avenir: pour une sociologie de la ruralité, Québec, Presses de l'Université du Québec, 1997.

${ }^{2}$ Voir son article "Social Implications of the agro-environmental policy in France and Europe" dans la revue Sociologia Ruralis, v. 35 , n. 2,1995 , p. 227-247. 
La question de la responsabilité environnementale des agriculteurs se trouve ainsi posée. L'élaboration d'un code de bonnes pratiques agricoles compatibles avec la protection de l'environnement peut-il être confiée aux premiers intéressés, les agriculteurs? L'approche autorégulatrice, postulant leur capacité d'édicter des règles et d'en assurer l'application, est-elle une utopie romantique qu'il faut oublier pour plutôt mettre en place un dispositif juridique contraignant permettant alors de parler d'une judiciarisation des pratiques agricoles? Certaines mesures, comme celles prises en Ontario, montreront que, à l'encontre de ce nous pensions nous-mêmes il y a peu de temps ${ }^{3}$, le pouvoir politicoadministratif peut parfois s'en remettre à une telle approche autorégulatrice qui fait passer la responsabilisation individuelle et l'éducation avant la répression et les sanctions. La création récente au Québec des "cluba agri-environementaux" réunissant un groupe d'agriculteurs familiaux qui se paient une partie de leurs besoins en expertise technique en agrienvironnement marque pour nous le glissement décisiif de cette agriculture familiale vers une agriculture durable où les agriculteurs s' auto-responsabilisent en matière de protection de l'environnement.

Dans le présent article, nous passerons en revue les principaux dispositifs agri-environnementaux aux États-Unis pour nous transporter ensuite au Canada, et plus particulièrement au Québec. Ce déplacement du regard sera facilité par l'existence du Plan nordaméricain de gestion de sauvagine qui couvre les deux pays. Finalement, après avoir rappelé l'importance du développement de l'agriculture durable en Amérique du Nord et aussi l'émergence de la notion de responsabilité environnementale, nous discuterons de la relation entre l'agriculture familiale et l'agriculture durable.
La version contemporaine du conservationnisme: le Conservation Reserve Program aux États-Unis

Aux États-Unis, la notion de conservation des ressources naturelles en agriculture remonte au fameux Dust Bowl des années trente où les grandes prairies fertiles étaient en train de devenir un désert sous l'effet de l'érosion éolienne conjuguée avec une variation climatique imprévue et des pratiques agricoles peu compatibles avec le maintien de la fertilité à long terme des sols. La question de l'érosion des sols est donc devenue, avec celle de la qualité de l'eau plus tard, centrale dans la problématique environnementale nord-américaine. Depuis cette époque jusqu'à aujourd'hui, une série de programmes a été mise en place pour retirer des terres de la production agricole, qu'on appelle aujourd'hui gel des terres. Ces programmes de retrait visaient deux buts simultanément, premièrement, la réduction de la perte d'humus dans les sols, soit la poursuite de l'objectif écologique de la durabilité de cette ressource, et deuxièmement, la réduction de la surproduction agricole, soit la poursuite de l'objectif économique de la durabilité économique de l'agriculture elle-même.

L'actuel Conservation Reserve Program (le CRP) qui est devenu avec le dernier Farm Bill (nom populaire du Food Security Act) de 1996 le ECARP, pour Environmental Conservation Acreage Reserve Program, trouve son origine dans ces programmes gouvernementaux de retraits des terres agricoles aux activités culturales, comme les National Grasslands sous la responsabilité du Service des Forêts de l'administration américaine ou la Soil Bank des années cinquante. En général, les agriculteurs qui retirent des terres à la production agricole dans ce cadre sont indemnisés par des fonds publics sur la

\footnotetext{
${ }^{3}$ Nous rendons compte de cette analyse dans un article polémique dansun grand quotidien du Québec, Le Devoir, publié à Montréal. Voir: "Urbs contre rurs. L'agriculture durable ou la judiciarisation des pratiques agro-environnementales", dans le quotidien Le Devoir (Montréal), 9 décembre 1994, p. A-11 (en collaboration avec Andrée Deschênes).
} 
base d'un calcul complexe du partage des coûts (le cost-sharing), ou plus précisément des pertes encourues par les fermiers. En fait, les montants sont proportionnels aux avantages escomptés pour l'environnement et aux économies budgétaires prévues. La mise en réserve (le set-aside comme version moderne de la jachère) de ces terres signifie généralement les remettre en prairie permanente, étant entendu que cette couverture herbagère pérenne est la meilleure manière de protéger la ressource agricole. La diversité biologique est ainsi protégée car l'écosystème de la prairie permanente mitée de quelques marais et d'une végétation arbustive est célébrée comme le milieu naturel par excellence pour la reproduction de la faune et de la flore. Mais cette vision d'une certaine forme d'agriculture compatible avec le maintien de la biodiversité est une idée neuve, un produit de la prise de conscience écologique des années soixante, éveillée aux États-Unis par des cris d'alarme comme Silent Spring de Rachel Carson.

Deux concepts caractérisent l'approche américaine de protection de l'environnement en agriculture: les dispositions de soutien conditionnel (cross compliance) et le ménagement des terres en "bon père de famille" (private stewardship). La cross compliance désigne les dispositions d'engagement conditionnel du Food Security Act de 1985, et reprise dans les versions ultérieures, loi plus connue sous le nom populaire de Farm Bill, en vertu desquelles les agriculteurs sont tenus d'adopter des plans de conservation approuvés pour pouvoir continuer à bénéficier des avantages financiers des programmes agricoles fédéraux s'ils exploitent des terres très sensibles à l'érosion. Et avec la fin de certains programmes suite aux nouveaux accords du GATT, on parle maintenant de soutien compensatoire.

La notion de private stewarship désigne la gestion écologiquement prudente des terres agricoles par ceux qui les possèdent. Mais plus de $50 \%$ du sol américain est possédé par des particuliers, et comme la propriété, c'est le droit d'user et d'abuser, cette notion introduit une philosophie de gestion ou d'usage d'un bien en "bon père de famille" comme il est écrit dans certains textes de loi. Pour traduire cette notion en français, les mots de gérance, de régie ou mieux d'intendance ne conviennent guère. Car il y a cette idée de se mettre volontairement au service d'une cause, de prendre soin de quelqu' un ou quelque chose, à l'image du travail du steward sur un vol d'avion. Il faut donc parler ici de "ménagement" des terres, selon la proposition de Christian Deverre qui reprend une vieille idée d'un pionnier de l'agronomie, Olivier de Serres.

Une multitude de recherches a été menée sur l'effet réel de ces programmes en terme d'impact sur l'environnement agricole. Les engagements contractuels concernant le retrait des terres menacées d'érosion de la production agricole sont limités à dix ans. Les effets sont variables selon la conjoncture de l'économie agricole d'ensemble. Par exemple, la réduction de la production de céréales dans les Prairies durant les années soixante-dix, attribuables à ces actions de protection a été largement annulée par une hausse des prix des céréales. Pendant que des terres étaient retirées de la production à un endroit avec l'aide des fonds publics, sur une autre parcelle peu éloignée, dans la même localité voire dans la même exploitation, on convertissait en production céréalière d'autres terres avec les généreuses aides publiques de soutien à la production agricole. D'où le constat désabusé de certains chercheurs ${ }^{4}$ sur les effets positifs de ce type d'intervention en terme de conservation, effets qui ne dureraient que le temps que durent les programmes étatiques.

Pourtant, d'autres économistes ont pu montrer, quelques années après la mise en route du $\mathrm{CRP}$, que celui-ci avait permis d'établir une couverture végétale permanente sur plus de dix millions d'hectares et que les avantages économiques de ce seul programme, en tenant compte de tous les "effets bénéfiques sur les ressources naturelles", se situent entre 3,4 et 11 milliards de dollars par année. Actuellement, un tiers

${ }^{4}$ RIBAUDO, M. O.; S. PIPER et al. “CRP: What economic benefits?”' dans Journal of Soil \& Water Conservation, v. 44, n. 5, p. 421 -425 . 
La question de la responsabilité environnementale des agriculteurs se trouve ainsi posée. L'élaboration d'un code de bonnes pratiques agricoles compatibles avec la protection de l'environnement peut-il être confiée aux premiers intéressés, les agriculteurs? L'approche autorégulatrice, postulant leur capacité d'édicter des règles et d'en assurer l'application, est-elle une utopie romantique qu'il faut oublier pour plutôt mettre en place un dispositif juridique contraignant permettant alors de parler d'une judiciarisation des pratiques agricoles? Certaines mesures, comme celles prises en Ontario, montreront que, à l'encontre de ce nous pensions nous-mêmes il y a peu de temps ${ }^{3}$, le pouvoir politicoadministratif peut parfois s'en remettre à une telle approche autorégulatrice qui fait passer la responsabilisation individuelle et l'éducation avant la répression et les sanctions. La création récente au Québec des "cluba agri-environementaux" réunissant un groupe d'agriculteurs familiaux qui se paient une partie de leurs besoins en expertise technique en agrienvironnement marque pour nous le glissement décisiif de cette agriculture familiale vers une agriculture durable où les agriculteurs s' auto-responsabilisent en matière de protection de l'environnement.

Dans le présent article, nous passerons en revue les principaux dispositifs agri-environnementaux aux États-Unis pour nous transporter ensuite au Canada, et plus particulièrement au Québec. Ce déplacement du regard sera facilité par l'existence du Plan nordaméricain de gestion de sauvagine qui couvre les deux pays. Finalement, après avoir rappelé l'importance du développement de l'agriculture durable en Amérique du Nord et aussi l'émergence de la notion de responsabilité environnementale, nous discuterons de la relation entre l'agriculture familiale et l'agriculture durable.
La version contemporaine du conservationnisme: le Conservation Reserve Program aux États-Unis

Aux États-Unis, la notion de conservation des ressources naturelles en agriculture remonte au fameux Dust Bowl des années trente où les grandes prairies fertiles étaient en train de devenir un désert sous l'effet de l'érosion éolienne conjuguée avec une variation climatique imprévue et des pratiques agricoles peu compatibles avec le maintien de la fertilité à long terme des sols. La question de l'érosion des sols est donc devenue, avec celle de la qualité de l'eau plus tard, centrale dans la problématique environnementale nord-américaine. Depuis cette époque jusqu'à aujourd'hui, une série de programmes a été mise en place pour retirer des terres de la production agricole, qu'on appelle aujourd'hui gel des terres. Ces programmes de retrait visaient deux buts simultanément, premièrement, la réduction de la perte d'humus dans les sols, soit la poursuite de l'objectif écologique de la durabilité de cette ressource, et deuxièmement, la réduction de la surproduction agricole, soit la poursuite de l'objectif économique de la durabilité économique de l'agriculture elle-même.

L'actuel Conservation Reserve Program (le CRP) qui est devenu avec le dernier Farm Bill (nom populaire du Food Security Act) de 1996 le ECARP, pour Environmental Conservation Acreage Reserve Program, trouve son origine dans ces programmes gouvernementaux de retraits des terres agricoles aux activités culturales, comme les National Grasslands sous la responsabilité du Service des Forêts de l'administration américaine ou la Soil Bank des années cinquante. En général, les agriculteurs qui retirent des terres à la production agricole dans ce cadre sont indemnisés par des fonds publics sur la

\footnotetext{
${ }^{3}$ Nous rendons compte de cette analyse dans un article polémique dansun grand quotidien du Québec, Le Devoir, publié à Montréal. Voir: "Urbs contre rurs. L'agriculture durable ou la judiciarisation des pratiques agro-environnementales", dans le quotidien Le Devoir (Montréal), 9 décembre 1994, p. A-11 (en collaboration avec Andrée Deschênes).
} 
base d'un calcul complexe du partage des coûts (le cost-sharing), ou plus précisément des pertes encourues par les fermiers. En fait, les montants sont proportionnels aux avantages escomptés pour l'environnement et aux économies budgétaires prévues. La mise en réserve (le set-aside comme version moderne de la jachère) de ces terres signifie généralement les remettre en prairie permanente, étant entendu que cette couverture herbagère pérenne est la meilleure manière de protéger la ressource agricole. La diversité biologique est ainsi protégée car l'écosystème de la prairie permanente mitée de quelques marais et d'une végétation arbustive est célébrée comme le milieu naturel par excellence pour la reproduction de la faune et de la flore. Mais cette vision d'une certaine forme d'agriculture compatible avec le maintien de la biodiversité est une idée neuve, un produit de la prise de conscience écologique des années soixante, éveillée aux États-Unis par des cris d'alarme comme Silent Spring de Rachel Carson.

Deux concepts caractérisent l'approche américaine de protection de l'environnement en agriculture: les dispositions de soutien conditionnel (cross compliance) et le ménagement des terres en "bon père de famille" (private stewardship). La cross compliance désigne les dispositions d'engagement conditionnel du Food Security Act de 1985, et reprise dans les versions ultérieures, loi plus connue sous le nom populaire de Farm Bill, en vertu desquelles les agriculteurs sont tenus d'adopter des plans de conservation approuvés pour pouvoir continuer à bénéficier des avantages financiers des programmes agricoles fédéraux s'ils exploitent des terres très sensibles à l'érosion. Et avec la fin de certains programmes suite aux nouveaux accords du GATT, on parle maintenant de soutien compensatoire.

La notion de private stewarship désigne la gestion écologiquement prudente des terres agricoles par ceux qui les possèdent. Mais plus de $50 \%$ du sol américain est possédé par des particuliers, et comme la propriété, c'est le droit d'user et d'abuser, cette notion introduit une philosophie de gestion ou d'usage d'un bien en "bon père de famille" comme il est écrit dans certains textes de loi. Pour traduire cette notion en français, les mots de gérance, de régie ou mieux d'intendance ne conviennent guère. Car il y a cette idée de se mettre volontairement au service d'une cause, de prendre soin de quelqu' un ou quelque chose, à l'image du travail du steward sur un vol d'avion. Il faut donc parler ici de "ménagement" des terres, selon la proposition de Christian Deverre qui reprend une vieille idée d'un pionnier de l'agronomie, Olivier de Serres.

Une multitude de recherches a été menée sur l'effet réel de ces programmes en terme d'impact sur l'environnement agricole. Les engagements contractuels concernant le retrait des terres menacées d'érosion de la production agricole sont limités à dix ans. Les effets sont variables selon la conjoncture de l'économie agricole d'ensemble. Par exemple, la réduction de la production de céréales dans les Prairies durant les années soixante-dix, attribuables à ces actions de protection a été largement annulée par une hausse des prix des céréales. Pendant que des terres étaient retirées de la production à un endroit avec l'aide des fonds publics, sur une autre parcelle peu éloignée, dans la même localité voire dans la même exploitation, on convertissait en production céréalière d'autres terres avec les généreuses aides publiques de soutien à la production agricole. D'où le constat désabusé de certains chercheurs ${ }^{4}$ sur les effets positifs de ce type d'intervention en terme de conservation, effets qui ne dureraient que le temps que durent les programmes étatiques.

Pourtant, d'autres économistes ont pu montrer, quelques années après la mise en route du CRP, que celui-ci avait permis d'établir une couverture végétale permanente sur plus de dix millions d'hectares et que les avantages économiques de ce seul programme, en tenant compte de tous les "effets bénéfiques sur les ressources naturelles", se situent entre 3,4 et 11 milliards de dollars par année. Actuellement, un tiers

${ }^{4}$ RIBAUDO, M. O.; S. PIPER et al. “CRP: What economic benefits?” dans Journal of Soil \& Water Conservation, v. 44, n. 5, p. 421 $-425$. 
des terres éligibles est protégée dans le cadre du CRP et les contrats nécessitent des déboursés de l'ordre de 1,4 milliards de dollars par année 5 . Les évaluations de l'OCDE sont fort positives car il serait plus facile d'assurer l'entretien des terres mis hors culture en les qualifiant de "réserves de conservation" avec le CRP. Il devient du devoir de l'agriculteur d'en prendre soin d'une manière qui augmente la qualité des paysages et contribue à la protection de la nature. Une pression sociale sensible s'exerce sur les agriculteurs afin d'instituer sur ces terres protégées des activités qui confortent leur valeur écologique.

Le CRP est aussi l'aboutissement du travail des mouvements écologistes modernes, et des vieilles associations conservationnistes comme le Sierra Club, Audubon Society ou la National Wildlife Federation, rassemblant des intérêts divers et constituant de puissants lobbies. Ces lobbies ont soudainement réalisé que la protection de la faune et la flore n'étaient pas possible sans un dialogue avec ceux qui possédaient plus de $50 \%$ du sol américain, c'est-à-dire les agriculteurs. Cela s'est fait autour des débats pour la passation du premier Farm Bill de 1985, alors appelée le Food Security Act, et qui définit le dispositif d'ensemble de la politique agricole américaine; à ce moment, est introduit le Title Conservation qui va créer le CRP.

L'objectif initial du CRP était de retirer de la production agricole 16 à 18 millions d'hectares de terres très sensibles à l'érosion (highly erodible cropland) en les convertissant en prairie ou en forêt. Pour les écologistes, l'inquiétude reste grande. On retrouve aux États-Unis 81 millions d'hectares de terres humides (Wetlands) dont la moitié a disparue par des aménagements de drainage et de mise en culture commerciale. Il s'agit d'un des écosystèmes les plus productifs au monde qui assure la reproduction de la moitié des espèces menacées de disparition. Les puissants lobbies de défense des canards rassemblent aussi des amateurs de chasse au canard qui voyaient ainsi leur loisir préféré disparaître à terme. Ironiquement, on pourrait que dire que le sort les canards d'Amérique dépend de ceux qui veulent, en définitive, leur faire la chasse. D'autres lobbies combattent avec les armes du système, l'argent et la propriété foncière. Par exemple, Ducks Illimited, une fondation privée, qui fait de grandes levées de fond dans le public et dont les avoirs se comptent en milliards de dollars, achète aux agriculteurs, au prix fort, des marais entiers, pour en assurer leur protection intégrale. Ce faisant, le maintien de ces milieux essentiels à la reproduction dune partie de la faune nord-américaine est aussi assuré.

\section{Le virage agri-environnemental du récent Farm Bill}

Contrairement aux craintes exprimées par les lobbies conservationnistes, le dernier Farm Bill renforce sensiblement le dispositif de protection de l'environnement en agriculture. Le Conservation Reserve Program est redéfini avec la mise en place de l'ECARP. Une fondation est aussi créée, la National Natural Resources Conservation Foundation, qui doit concerter les actions de protection de l'environnement agricole des différents niveaux de gouvernements, initier desrechercheset des expérimentations avec les agriculteurs, soutenir des programmes de vulgarisation sur les techniques agricoles plus respectueuses de l'environnement. De plus, dans les sections intitulées Environmental quality incentives program, Conservation of private grazing land et Wetlands Reserve Program, on retrouve des dispositions qui diffèrent sensiblement de l'approche classique de préservation de la ressource par la conversion des terres fragiles en une sorte de prairie permanente herbacée, voire même arbustive. Un soutien technique et financier est offert aux agriculteurs

${ }^{5}$ NELLIS, Duane M. "Agricultural Externalities and the Environment in the United States" dans I. R BOWLER; C. R. BRYANT; M. D. NELLIS, Contemporary rural systems in transition, Vol 1, Agriculture and Environnement, Wallingford (UK), C.A.B. International, 1992, p. 131-141. 
qui vont mettre en place, sur leur exploitation, un conservation system, c'est-à-dire un ensemble de pratiques agricoles compatibles avec une agriculture respectueuse de l'environnement, selon un cahier des charges établi en fonction des conditions propres à chaque exploitation par les techniciens du National Resources Conservation Service.

L'introduction du concept de conservation system dans le dernier Farm Bill nous n'est pas rappeller la notion de "plans de développement durables" apparue dans les récentes versions des mesures agri-environnementales européennes. La notion est définie comme l'ensemble des mesures de conservation et des pratiques agricoles approuvées et mises en oeuvre par un producteur sur des sols menacés d'érosion et qui vont se traduire par une réduction de cette érosion au meilleur coût possible. Pour le choix de ces mesures et de ces pratiques, on doit recourir au guide technique du Service de conservation des ressources naturelles de l'USDA en tenant compte de ces standards mais aussi des conditions locales. On introduit aussi dans le récent Farm Bill la notion de land management practice qui est définie comme les différentes formes de gestion des terres touchant la fertilisation chimique, l'épandage des fumiers, l'usage des pesticides, le travail du sol, etc... identifiées par le Secrétaire de l'USDA comme étant compatibles avec la protection, au meilleur coût possible, des sols, de l'eau et des autres ressources reliées à l'agriculture.

Dans le cas américain, on retrouve aussi la notion d'éligibilité et ce programme concerne évidemment les environnementally sensitive fields déjà bien identifiés. Par ailleurs, les mesures de soutien à la conversion des terres en prairies permanentes, ce qu'on appelle dans le texte du Farm Bill les nonfederally owned rangeland, pastureland, grazed forest land and hay land, ne sont pas sans rappeler, une fois de plus, certaines mesures agri-environnementales européennes.
D'autre part, la notion de partage des coûts entre les agriculteurs et le reste de la société est réaffirmée. Les sommes provenant de fonds publics que peut recevoir une même exploitation qui participe à différents programmes pouvant être considérables, le législateur américain a établi une limite supérieure, de 50,000 dollars, par exploitation agricole. Cette somme est substantielle car plusieurs petites fermes présentent un chiffre d'affaires bien inférieur à cette limite. Le texte du Farm Bill est particulièrement détaillée, explicite, avec l'énoncé d'une longue liste de considérants pour justifier des dépenses de fonds publics chez des propriétaires privés; il pourrait ainsi donner prise à une véritable analyse du discours. On prend toujours la peine d'affirmer que les dépenses en faveur d'une entreprise privée vont générer des avantages pour tous les citoyens américains.

Par exemple, on affirme "que les agriculteurs ont fait des efforts remarquables pour protéger l'environnement et les ressources agricoles des ÉtatsUnis au cours de la dernière décennie non pas seulement en réponse aux programmes du gouvernement Fédéral mais aussi en suivant leur "esprit de ménagement» (spirit of stewardship) et l'application de technologiques appropriées". Or, d'après les nombreuses recherches sur le sujet ${ }^{6}$, les agriculteurs ont été très sensibles aux subventions, aux primes. Le texte final du Farm Bill reflète bien le rapport de forces entre les différents lobbies agricoles et environnementaux. Dans ce cas, il s'agit de rechercher l'adhésion des agriculteurs en les présentant, à leurs propres yeux comme à ceux du grand public, comme des citoyens responsables qui sont déjà sérieusement engagés dans la préservation de l'environnement naturel. Plus loin, on laisse entendre que les agriculteurs ne sont pas aussi responsables, qu'ils ne pourront y arriver tout seuls, et qu'il faut donc une intervention publique en faveur de ces propriétaires privés.

\footnotetext{
${ }^{6}$ Une revue scientifique comme le Journal of Soil Science and Water Management semble d'ailleurs avoir fait de cette question un thème majeur de publication.
} 
Ces nombreux "considérants" qui émaillent le texte du Farm Bill sont autant des énoncés de faits attestés, comme "les agriculteurs et les propriétaires de ranches possèdent la moitié du sol des États-Unis" ou "les prairies herbagées (private grazing lands) constituent le plus grand habitat pour la faune (wildlife habitat) aux États-Unis" que des propositions incantatoires proches du discours idéologique telles que "la mise en oeuvre de l'ECARP devrait réconcilier la productivité et la profitabilité avec la protection et l'amélioration de l'environnement".

\section{Le Plan nord-américain de gestion de la sauvagine}

$\mathrm{Au}$ cours des années quatre-vingt, un très important programme de protection des terres humides dans les Prairies canado-américaines a été mis sur pieds par les agences publiques et privées de protection de l'environnement. Le North American Waterfowl Management Plan dépense 1.5 milliards de dollars par année pour offrir des aides aux agriculteurs pour ne pas cultiver ces milliers de petits "potholes" qui sont autant de petites parcelles de terres humides fort utiles pour la reproduction de la sauvagine certes mais aussi de 168 autres espèces fauniques d'Amérique du Nord. En vertu de ce programme, 160,000 hectares ont été réservés à l'habitat de la sauvagine dans la seule région des Prairies canadiennes; et plus de 300,000 hectares ont été consacrés à l'habitat faunique. Ce programme permet, dans certains cas, le maintien des activités agricoles compatibles avec la protection de la faune sur ces terres protégées.

Tous les observateurs ne tarissent pas d'éloge sur un tel programme qui fait l'affaire groupes environnementaux et des agriculteurs qui, en acceptant de préserver ces marais (auparavant, par des actions drainage, on les faisait disparaître), touchent une rémunération pour les "services écologiques" rendus à toute la société. On a aussi démontré que les sommes investis par les pouvoirs publics en vertu de ce programme étaient totalement récupérées (et même trois fois plus) par les économies sur les subventions publiques d'aide à la production agricole qui auraient été versées aux producteurs si ces terres étaient cultivées.

Un économiste, parlant de ce Plan nord-américain de gestion de la sauvagine, a ainsi dit que dans ce cas "ce sont les canards qui paient la facture". Il laissait entendre que, par les agences publiques et privées de protection de l'environnement interposées, les canards étaient capables de payer le prix qu'il faut pour maintenir leur habitat. Évidemment, ce sont pas toutes les espèces qui jouissent d'une pareille sollicitude dans le public. Le canard est devenu une sorte de symbole mythique dans la défense de l'environnement à l'américaine. Comme si la présence de ces oiseaux rappelait que des éléments de la nature avaient été préservés dans un environnement hautement transformé par l'homme... alors que dans certain cas, on peut se demander si on n'est pas en train de reconstruire, sur une grande échelle, une nature "sauvage" comme dans le cas des grands parcs urbains qui sont inconcevables sans les canards et toute une faune ailée.

Chaque année sur les rives de l'Estuaire du Saint-Laurent au Québec, les oiseaux migrateurs s'arrêtent pour s' alimenter sur quelques riches marais le long de ses berges. Mais les oies blanches ont la faucheuse habitude de s'en prendre aux champs cultivés par les agriculteurs à proximité de ces marais. Des écologistes ont prétendu que les agriculteurs auraient transformés une bonne partie de ces marais en terres agricoles... mais où finit la terre et commence le marais? Laissons ces disputes à ceux qui les animent. Toutefois, à chaque année, trois à quatre cents exploitations reçoivent du gouvernement

${ }^{7}$ Cette notion de services écologiques que peuvent rendre les agriculteurs et qui peuvent être financés par le marché ou toute la société a été énoncé par un économiste canadien, Robert D. SOPUCK dans un rapport de la Table ronde nationale sur l'environnement et l'économie en 1993.

${ }^{8}$ Robert D. SOPUCK. Idem, p. 49. 
près d'un million de dollars pour les dommages subis par le passage des oies blanches trop gourmandes. D'une certaine manière, on peut donc dire que ces oiseaux migrateurs sont riches et qu'ils ont les moyens de payer.

Je prends soin de ma terre: la transition de l'agriculture québécoise aux modes de production durable

Au Québec, on parle des pratiques agroenvironnementales, ou des modes de production durable, pour désigner des pratiques agricoles respectueuses de l'environnement. Les mouvements écologistes s'en prennent volontiers au secteur agricole accusé, par exemple, de déverser $40 \%$ des charges polluantes dans les cours d'eau du Québec. Les agriculteurs essaient tant bien que mal de se défendre de ces attaques du mouvement écologiste qui trouve, avec le développement rapide de l'industrie porcine, motif à inquiétudes pour la santé publique. De là, leur requête pour une loi sur le droit de produire qui fait d'ailleurs penser au droit de polluer de l'industrie.

Les agriculteurs québécois sont d'autant plus gênés par ces accusations que la production laitière, selon le système agraire classique de "polycultureélevage", apparaît d'emblée comme un système productif plus proche d'une agriculture raisonnée respectueuse de l'environnement. Toutefois, comme ailleurs, on a assisté à une diminution des prairies avec le développement des grandes cultures commerciales telle que le maïs; mais celles-ci ne couvrent que $23 \%$ des terres. La proximité des meilleures terres agricoles des grands centres urbains, ayant tendance à s'étaler fortement, rend la cohabitation des activités urbaines et agricoles souvent difficile. Par ailleurs, ces critiques du mouvement écologiste qui, sur la base de quelques cas réels, définit d'emblée cette agriculture comme agriculture polluante, reposent sur une ignorance étonnante et des efforts de transition vers l'agriculture durable réalisés au Québec et des attitudes des agriculteurs face à l'environnement.

La protection de l'environnement prend ici l'allure d'une approche sectorielle de protection ou de conservation des diverses ressources agricoles, dont les principales sont le sol et l'eau. Comme moins de $4 \%$ du sol québécois est propre à la culture et comme les meilleures terres sont menacées par l'expansion urbaine, la Loi sur la protection du territoire agricole, l'une des plus coercitives qui soient, a permis de protéger efficacement cette ressource, du moins de d'autres usages. Et comme plus du tiers des terres sont des prairies de plantes fourragères, les problèmes d'érosion, si typiques de l'agriculture américaine, y sont donc beaucoup moins importants. On raconte ainsi que "la régie de ces sols favorise l' amélioration de leur structure et le maintien de la matière organique vu le couvert végétal et le lacis racinaire permanents (...) qu'ils sont donc très peu affectés par l'érosion, la perte de matière organique, le bris des agrégats et le compactage attribuables aux cultures et aux passages des instruments et de l'équipement lourd et (...) que leur fertilisation est assure majoritairement par des engrais de ferme appliqués à la suite de la coupe du foin." 9

Par ailleurs, la question de l'eau y prend aussi une coloration singulière. Ce territoire est fortement arrosé par les pluies et la fonte des neiges. Depuis plus d'un siècle, on a fait d'importants travaux de drainage (sur 500,000 ha) pour profiter pleinement d'une saison de végétation sans gel qui varie de 60 à 160 jours selon les régions. Le drainage aidant, la détérioration des cours d'eau peut se faire de deux manières: la pollution des sources ponctuelles (déversement de purin par exemple) et celle de sources diffuses (lessivage et ruissellement de surface entraînant des pesticides dans la nappe phréatique).

Au premier rang de ces pratiques agri-environnementales dans lesquelles semblent s'engager assez

${ }^{9}$ Anne-Marie CHRISTEN. "Le bilan des pratiques agroenvironnementales" dans L'Action Nationale, v.86, n.1, juin 1986, p. 65 (L'auteur est une agronome au service de l'Union des producteurs agricoles du Québec). 
résolument l'agriculture québécoise, il faut parler de la gestion des engrais de ferme avec ce qu'on appelle les PGFI, pour plan global de fertilisation intégrée. Il s'agit d'un plan de fertilisation qui valorise au mieux les ressources propres de la ferme. La mise en oeuvre d'un tel PGFI suppose généralement l'adoption de d'autres pratiques agricoles plus compatibles avec le respect de l'environnement. Si les PGFI constituent une bonne réponse aux préoccupations environnementales, l'installation de plusieurs très grosses porcheries dans des bassinsversants sensibles n'est pas sans inquiéter une opinion publique alertée par le discours souvent apocalyptique des écologistes. La mise en oeuvre des PGFI au Québec relève de ce qu'on appelle au Canada la méthode du Plan agro-environnemental qui consiste en la rédaction et l'application d'un cahier des charges individualisé à chaque exploitation des pratiques agricoles respectueuses de l'environnement; toutefois, l'application du programme reste volontaire.

On assiste alors à une escalade de la réglementation sur les procédures d'épandage des purins qui n'est pas sans nous rappeler une sorte de judiciarisation des pratiques agricoles. Aussi, pour les agriculteurs québécois, l'enjeu actuel, c'est de sortir de logique de la réglementation et de la judiciarisation, pour aller plutôt vers une approche d'autorégulation, ou de coopération volontaire avec les différents partenaires intéressés. Ici, on n'en est pas à un paradoxe près. Alors que l'écologie nécessite d'agir localement, la capacité d'action locale de l'instance politique locale est telle qu'elle peut interdire telle ou telle activité agricole sur son territoire. Les lobbies environnementalistes ont d'ailleurs compris cette opportunité d'action.

Dans ce contexte, on comprend mieux que pour les agriculteurs, la revendication d'un droit de produire (évidemment limité aux terres "zonées" agricoles au sens de la Loi de protection du territoire agricole) n'est en rien un droit de polluer, mais seulement la reconnaissance du droit d'exercer librement une activité sans subir quotidiennement la menace de recevoir des avis et des accusations en vertu de leur responsabilité environnementale. Car la protection que cette loi accorde consiste à déplacer le fardeau de la preuve de l'agriculteur au plaignant; celui doit prouver que l'agriculteur ne s'est pas conformé aux normes environnementales. Mais pour les agriculteurs, cela est important car avec une telle loi sur le droit de produire, ils se sentiraient moins des criminels en puissance et la société prendrait acte de l'importance de l'agriculture dans la nécessaire revitalisation du monde rural.

En 1998, s'est tenu au Québec un important sommet de tous les partenaires socio-économiques du monde agricole. De manière assez surprenante, l'idée d'une auto-responsabilisation des agriculteurs, et c'est le concept même qui est indiqué dans la documentation gouvernementale, est devenue le point de vue officiel de l'État qui apparaissait pourtant attaché à la vieille approche réglementaire et judiciaire. Avec la mise ne place de clubs agrienvironnementaux pour l'encadrement technique en agriculture durable, la province de Québec s'est engagée, comme l'Ontario et la Colombie britannique leurs "Environmental Farm Plan", résolument dans cette approche autorégulératrice de l'environnement en agriculture.

\section{La progression de l'agriculture durable en Amérique du Nord}

La notion d'agriculture durable progresse plutôt rapidement en Amérique. Un bon exemple, c'est la révolution actuelle en agronomie ${ }^{10}$, ou plutôt dans une de ses branches constitutives, la science des sols. Les ténors de ce savoir disciplinaire ont longtemps

\footnotetext{
${ }^{10}$ Dans le cas québécois, cette révolution, typique des révolution scientifique selon le modèle de Kuhn, est bien décrite dans une recherche récente. Voir: Stéphane CASTONGUAY. "Conservation des ressources et changement scientifique agronomie au Québec" dans Recherches sociographiques, v.36, n.3, 1995, p. 479-526 (Revue publiée par le Département de sociologie de l'Université Laval à Québec ISSN 0034-1282).
} 
récusé la pertinence de l'analyse microbienne de sols réclamée par l'agriculture biologique (Nourrir le sol qui nourrit la plante....) au profit de l'analyse minérale permettant un diagnostic et une ordonnance sur les fertilisants chimiques à apporter au sol en fonction de la culture envisagée. Dans le contexte québécois, on parle aussi très largement de la conversion à l'agriculture biologie ou à l'agriculture durable. Cela entraîne une forte demande de formation sur mesure à l'adresse des instituts agronomiques ou de stations expérimentales agricoles qui tournaient à vide depuis un certain temps. Ils se trouvent ainsi une nouvelle vocation qui les met à l'abri des tentatives de fermetures d'une administration qui vit aux rythmes des compressions budgétaires. Cela veut aussi dire que la demande vient des exploitants modernisés actuels, et non de groupes d'agriculteurs néo-ruraux marginaux. Il s'agit ici d'un phénomène étonnant qui reste encore à expliquer $^{11}$.

Aux États-Unis, une grande firme d'agrofourniture, Monsanto, le fabricant du célèbre pesticide Round-up, retrouve grâce auprès des lobbies environnementalistes prêts à lui attribuer un prix écologiste important, à cause de ses investissements massifs dans la recherche sur les biotechnologies douces, qui a aussi le mérite de créer des emplois pour des écologistes convaincus. Ici, on joue sur une corde sensible de l'imaginaire américain, Silent Spring révélait les méfaits du DDT maintenant interdit (banni d'usage seulement, ce qui n'empêche pas sa fabrication à destination de pays plus laxistes en matière de législations sur les produits dangereux), et encore aujourd'hui, une agriculture biologique, c'est une chemical free agriculture, ou comme on dit maintenant, une LISA pour Low Input Sustainable Agriculture. Et là, on est pas loin du modèle proposé actuellement par le syndicalisme agricole français sous la notion d'agriculture raisonnée respectueuse de l'environnement'2. Mais actuellement, on pourrait dire que les agriculteurs nord-américains voudraient bien, qu'en échange de leur devoir de conserver les ressources naturelles, qu'on leur accorde un véritable droit de produire.

\section{L'émergence du concept de responsabilité environnementale}

Agriculture et Agroalimentaire Canada, par son Bureau de l'environnement, a rendu publique récemment une étude ${ }^{13}$ rédigée par des spécialistes du droit de l'environnement et qui illustre bien notre hypothèse de la judiciarisation des pratiques agricoles dans le contexte canadien et qui est abordée ici par sa conséquence logique, soit l'émergence de la notion de responsabilité environnementale. La situation est telle qu'un propriétaire de terrain soupçonné par exemple d'y avoir enfoui illégalement des matières dangereuses peut se voir obligé par les tribunaux de remettre en état les lieux. Les organismes publics et privés qui prêtent de l'argent aux entreprises agricoles exigent de plus en plus des évaluations environnementales de peur voir leur créancier devenir insolvable. Car le propriétaire actuel, en vertu de droit canadien, est responsable pour les méfaits, même inconnus de lui, des propriétaires antérieurs.

Selon certains avocats spécialisés dans ce genre de cause, l'activité agricole a été généralement ex-

${ }^{11}$ Dans des enquêtes que nous réalisé auprès des producteurs agricoles du Québec, un agriculteur sur deux pense que dans l'avenir la conversion sera inévitable... autant s'y préparer tout de suite. Contrairement à l'opinion de certains écologistes, ils ne sentent guère des pollueurs mais plutôt comme des victimes la pollution; il est vrai que plusieurs de ces exploitants possèdent des érablières dont on pensait qu'elles se mourraient à cause des pluies acides... alors qu'il s'agissait d'un stress hydrique. Il s'agit ici d'un cas typique où les sciences écologistes, pressées par l'opinion publique et les décideurs, ont avancé une explication, largement admise par l'opinion parce que cautionnée par la science, qui était totalement erronée.

${ }^{12}$ D'après des informations du FARRE, le Forum pour une agriculture raisonnée et respectueuse de l'environnement. Concernant les pesticides et les engrais de synthèse, leur devise est: autant qu'il en faut mais le moins possible.

${ }^{13}$ Morrie PAUL. La responsabilité en matière d'environnement dans le secteur agricole, Ottawa, Bureau de l'Environnement de Agriculture et Agroalimentaire Canada, 1995, 54 p. 
clue des dispositifs juridiques qui concernent la responsabilité environnementale en matière de pollution. Le principe du pollueur-payeur a fait son chemin dans le domaine industriel bien que souvent ce soit la collectivité qui devient le payeur. Les entreprises connaissent généralement très bien jusqu'où va leur droit de polluer et les normes prescrites dans les textes de lois sont souvent issues d'une négociation sociale entre le monde industriel et les "lobbies" environnementaux. En agriculture, ce n'est évidemment pas le cas, à part le fait que les entreprises agricoles sont soumises, comme les autres citoyens ordinaires ou corporatifs, aux différentes lois sur la pollution ou la gestion des déchets dangereux. On peut alors se demander s'il ne serait pas avantageux pour l'agriculture de se doter d'un encadrement juridique, soit ce qu'on pourrait appeler un droit de l'exploitation agricole, ou comme on dit au Québec, un "droit de produire".

Plusieurs États américains et certaines provinces canadiennes se sont donnés un tel dispositif légal. Mais les spécialistes du droit de l'environnement semblent divisés sur la question à cause, en autre, de complexité de l'activité agricole et donc la difficulté de diagnostiquer ce qui constitue, pour une exploitation agricole qui est toujours un écosystème unique, un préjudice à l'environnement méritant une sanction prescrite par la loi. À moins de suivre la pente sans fin de la codification des "bonnes" pratiques agricoles au sens d'une loi de l'exploitation agricole, soit ce que nous avons appelé la judiciarisation de ces pratiques.

La responsabilité environnementale en agriculture, d'après les agriculteurs québécois, devrait être une responsabilité collective, et non la seule responsabilité des exploitants dont on assume qu'ils en ont toutefois une part. On déclare ainsi que "le secteur agricole n'a pas eu sa juste part des investissements publics pour aider les producteurs agricoles dans leurs efforts de dépollution (...) et que la pollution d'origine agricole n'est toujours pas considérée comme une responsabilité collective, alors qu'on a notamment investi des milliards de dollars dans la dépollution des eaux usées urbaines" ${ }^{\prime \prime}$. Un tel propos est intéressant à plus d'un titre. Par exemple, il sous-tend implicitement que l'agriculture est une forme de service public, ce qui selon une certaine vue des choses, fait sens en regard de l'énorme encadrement étatique de cette activité. D' autre part, on pourrait penser qu'il met sur le même plan des activités du domaine privée de la production agricole et celle du domaine public de la consommation urbaine générant ces eaux usées. Mais, là aussi, il y plusieurs opérateurs d'entreprises privées qui contribuent aussi à la formation du volume global d'eaux urbaines usées. Ces derniers arrivent ainsi à externaliser les coûts et internaliser les profits, selon la logique de toute entreprise... que ce syndicat agricole reprend à son compte, projetant alors l'image inverse à celle évoquée plus haut, soit celle de l'entrepreneur avisé.

Du côté européen, les "travaux sur les mesures agri-environnemtales" ont mis en évidence la dimension de la contractualisation dans la procédure et qui implique une reconnaissance nouvelle de l'exploitant agricole comme citoyen de plein droit, si on peut dire. Par ailleurs, on peut aussi se demander si cette citoyenneté retrouvée ne fait pas trop écho à une plus prosaique chasse aux primes, une pratique bien connue des agriculteurs. Dans ce cas, nous l'avons dit, l'agriculteur américain n'est pas insensible aux primes, relativement généreuses, des programmes agri-environnementaux. Mais là où la différence est plus notable, c'est sur la citoyenneté des agriculteurs. Les concepts centraux du dispositif américain, comme la cross compliance et le private stewardship sont l'expression même de l'agriculteur reconnu comme citoyen... et on touche ici un des mythes fondateurs de la société américaine ellemême. Mais le corollaire de la citoyenneté, c'est la responsabilité; pas étonnant alors de voir apparaître dans ce terreau le débat actuel sur la responsabilité environnemtale des agriculteurs.

\footnotetext{
${ }^{14}$ Tiré de Déclaration de l'UPA sur l'environnement dans La Terre de Chez Nous (Longueuil, Canada), 11-17 avril 1996, p. 4.
} 
Sur la dynamique de la ferme familiale face à l'environnement

Selon une analyse fort répandue ici, comme ailleurs, on assisterait à la lente mais irréversible évolution des fermes familiales en entreprises industrielles. Pourtant, pour plusieurs écologistes, les fermes artisanales seraient un modèle plus intéressant pour les travailleurs de la terre comme les consommateurs urbains; de plus, il s'agirait d'un modèle d'exploitation agricole susceptible de faire une gestion écologiquement intelligente des ressources agroécologiques.

Une idée largement répandue est celle qui consiste à penser que si l'agriculture écologique doit s'imposer, cela se fera nécessairement en changeant le mode de produire, aujourd'hui dominé par des grandes unités industrielles, pour revenir à des petites unités de type familial. Une telle conception pose plusieurs questions sociologiquement intéressantes sur le changement social et sa dynamique. Nous prétendons plutôt que l'agriculture biologique, écologique, ou durable, nécessite, pour se déployer une forme sociale d'organisation de la production spécifique, en l'occurrence la ferme familiale, davantage habilitée à mettre en oeuvre un tel système productif agricole.

Cela revient à dire que l'on considère que les systèmes productifs agricoles ne sont pas invariants des modes d'organisation de la production (forme de propriété et de transmission de la terre, organisation du travail, systèmes culturaux, etc...). Or, cette question mérite quelques observations tellement elle est lourde de conséquences sur l'analyse sociologique du changement social en regard des transformations techniques.

L'idée d'une indépendance entre le mode de produire en agriculture et les systèmes productifs agricoles va à l'encontre de la théorie des "farming systems" qui tient la forme sociale d'organisation de la production comme une dimension importante et à prendre en compte dans le fonctionnement d'un système productif agricole donné. Par ailleurs, la tradition marxiste nous a longtemps habitué à voir les choses de cette manière. On se rappelle les analyses classiques de Marx de la petite production parcellaire et la grande exploitation, seule capable d'intégrer les progrès techniques et donc la seule capable de survivre dans un capitalisme à maturité et dans le socialisme. On connait la suite de l'histoire... plusieurs grandes exploitations de la fin du XIXe siècle, comme les dinosaures, sont disparues. Et c'est la ferme familiale, modernisée, mécanisée, capitalisée, productive, intensive, qui a su trouver les moyens d'adopter très rapidement les innovations techniques lorsqu'on a eu la brillante idée de les mettre à leur portée (par exemple, le petit tracteur Ford en Amérique, adapté à la taille de la ferme et à l'épaisseur du portefeuille de son propriétaire).

Ce bref rappel théorique et historique montre que l'hypothèse marxiste du lien nécessaire entre la forme sociale d'organisation de la production agricole et le système technique n'est pas aussi évident. Et s'il n'y a pas nécessairement un déterminant de la technique, des systèmes productifs agricoles, sur la forme sociale de l'exploitation, cela revient à dire, contrairement à une idée passablement répandue et qui fait sens sous plusieurs rapports, que l'éventuelle généralisation de l'agriculture écologique ou durable ne générera pas d'emblée une consolidation de l'exploitation agricole familiale telle qu'on la connaît. Si celle-ci est toujours omniprésente dans les agricultures modernes des pays du Nord, même si on annonce trop souvent sa transformation subreptice en petite entreprise capitaliste, elle pourra continuer à consolider sa présence dans la mesure où on peut faire l'hypothèse qu'elle possède des atouts spécifiques pour réaliser la "conversion" ou la "reconversion" à l'agriculture durable.

Nos analyses sociologiques du phénomène vont devoir s'ajuster sensiblement à une réalité que peu de monde ont pu prédire il y a une quinzaine d'années. En effet, à cette époque, il était plus courant d'associer l'agriculture écologique à une triple marginalité ${ }^{15}$ : une agriculture marginale pratiquée

${ }^{15}$ Ce que nous avons fait nous-mêmes. Voir: BRUNO, Jean. "L'agriculture écologique" dans Agriculture et développement dans l'Est du Québec, Sillery, Presses de l'Université du Québec, 1985. 
par des agriculteurs marginaux (néo-ruraux) dans des régions agricoles en voie de marginalisation.

Selon le modèle d'interprétation esquissé ici, la généralisation de l'agriculture durable ne signifie pas automatiquement la consolidation de l'exploitation agricole familiale même si la ferme familiale est dotée d'une grande capacité d'adaptation aux diverses conjonctures socio-politiques et technico-économiques qui la rendent particulièrement apte à réussir cette nouvelle adaptation.

\section{Conclusion: sur la notion de "transition" ou "conversion" à l'agriculture durable}

L'exploitation agricole familiale a su démontrer une extraordinaire plasticité aux diverses conjonctures économiques, techniques et politiques. Aussi, notre opinion est que le développement d'une agriculture durable, aujourd'hui à l'agenda sociopolitique de la plupart des agricultures nationales, se fera d'autant plus facilement qu'on laissera les exploitations agricoles familiales conventionnelles opérer une transition vers l'agriculture écologique, comme on disait hier, l'agriculture durable comme on dit aujourd'hui, et l'agriculture intégrée comme on dira peut-être demain.

Si les fermes familiales sont restées une composante essentielle de la structure sociale de nos différentes agricultures, ce n'est pas par un souci de préservation du patrimoine mais c'est bien parce qu'elles ont répondu avec une efficience insoupçonnée aux grands impératifs de cette politique agricole moderne: la sécurité alimentaire et une alimentation à bon marché (la cheap food policy).

Cette capacité d'adaptation va jouer en leur faveur dans le processus de transition vers l'agriculture durable. Si on prend en considération ce qu'est le modèle de l'agriculture durable, c'est là qu'on peut voir que l'agriculture familiale a toujours été plus proche du modèle et qu'elle peut donc y revenir ou s'en rapprocher plus aisément. Selon notre recension de la littérature sur l'agriculture durable, quelques idées-forces peuvent la définir.

La transition vers l'agriculture écologique ne signifie pas le retour à une agriculture traditionnelle qui aurait été un âge d'or écologique. Un premier mythe est ainsi détruit. Un second mythe est aussi en train d'être détruit, soit celui de la non-viabilité économique de l'agriculture écologique; il fait écho à un grand mythe agronomique de notre temps, à savoir que l'intensification est la seule voie de salut sur le plan économique. Il s'en suit que l'extensification n'est pas nécessairement une pratique écologique.

Là où la jonction peut se faire plus facilement entre agriculture familiale et agriculture durable, entendu au sens que nous avons donné à ces notions plus haut, c'est sur la question de la gestion des ressources naturelles. Le sociologue français Marcel Jollivet ${ }^{16} \mathrm{a}$ bien montré le processus qui fait que dans le passage à la rationalité marchande, la validation sociale de l' agriculture se mesure à sa capacité à livrer des marchandises au marché. Dans ce cadre, un partie importante du travail social agricole devient un travail invisible, tenue pour nulle et non avenue par le marché; il s'agit évidemment de ce travail qui concourt au maintien de la fertilité naturelle des sols, et plus largement pourrions-nous dire, à la préservation des propriétés de l'outil de production que constitue une exploitation agricole.

La ferme familiale, avec la pratique d'une gestion patrimoniale des ressources naturelles, s'avère ainsi capable de prendre en considération les principaux impératifs écologiques, ce que ne pourraient pas faire les autres modes de production productivistes ou capitalistes. Mais ce n'est pas nécessairement un avantage absolu; si le marché accepte un jour d'incorporer dans la structure des prix les coûts écologiques, si on peut dire, la production des denrées agricoles primaires pourrait intéresser le grand capital. Donc l'évolution prochaine de l'agriculture pourrait se faire de manière

${ }^{16}$ Marcel JOLLIVET. "Rente foncière et gestion des ressources naturelles", Études rurales, n. 71/72, 1978, p. 257-274; voir aussi M. JOLLIVET (dir.). Pour une agriculture diversifiée, Paris, L'Harmattan, 1988 et sous la direction de M. JOLLIVET et N. MATHIEU, Du rural à l'environnement, Paris, L'Harmattan, 1989. 


\section{L'AGRICULTURE DURABLE}

\section{L'agriculture durable est un mode de production agricole:}

- assurant la qualité des produits et limitant les risques pour la santé humaine;

- respectueux de l'environnement et préservant les ressources naturelles;

- économiquement rentable;

- et permettant une bonne intégration sociale des personnes intervenant dans le processus de production et de transformation.

Selon le point de vue où on se place, on insistera plus sur:

- la production des matières premières agricoles (économistes),

- la gestion de l'environnement (écologistes)

- le maintien de la qualité de vie en milieu rural (sociologues).

Une agriculture durable est une agriculture:

- soutenable écologiquement

- efficiente économiquement

- équitable socialement

- supportable sociologiquement

invariante à la question écologique, sa forme sociale se dessinant à partir d'autres enjeux. En clair, il est bien possible que l'agriculture durable se présente selon des formes différentes selon les contextes sociohistoriques.

Mais il reste un autre enjeu de l'agriculture moderne, soit le découplage entre l'agriculture et le territoire qui, encore là, tend à valider la thèse de la supériorité de l'agriculture familiale dans la dynamique socio-historique. Une des fonctions historiques de l'agriculture aura été l'occupation du territoire. L'absence ou la faible logique marchande qui présidait à sa validation sociale autorisait son implantation dans des espaces où les contraintes écologiques étaient compensées par un plus grand travail humain pour aboutir à une production assurant la subsistance d'une famille.

Cette fonction centrale de l'agriculture dans l'occupation du territoire, fondant les identités nationales, a été reconnu tardivement et constitue un autre sous-produit de l'activité agricole fourni gratuitement à l'ensemble de la société. L'agriculture traditionnelle non encore soumise à la rationalité marchande, et même certaines formes d'agriculture familiale, ont donc assuré le lien avec une occupation du sol légitimant la prétention à la souveraineté nationale sur un territoire donné. Marie-Élizabeth Chassagne dans un livre intitulé Agriculture et territoire. Les mamelles de la France. a bien illustré ce couplage agriculture - territoire et surtout les conséquences d'un éventuel découplage. Une agriculture durable devra nécessairement se bâtir à partir de la reconnaissance sociale de cette fonction trop longtemps ignorée de l'agriculture, soit l'occupation du territoire national, et on peut imaginer que cela lui assurera une base solide pour lui permettre de durer de manière plus indépendante des aléas des conjonctures tant écologiques que socio-politiques. 


\section{RÉFÉRENCES}

BOWLER, I. R.; C. R. BRYANT; M. D. NELLIS, (eds.). Contempory Rural systems in Transition: Economy and Society, Oxford (UK), CAB International, 1992 Vol 1 Agriculture and Environment et Vol 2 - Economy and Society.

BRYANT, Christopher R.; Claude MAROIS (eds.). The sustainability of rural systems: proceedings. First meeting of the IGU Study Group on the Sustainability of Rural Systems, Montréal, Université de Montréal, Département de géographie, 1995.

CASTONGUAY, Stéphane. "Conservation des ressources et changement scientifique agronomie au Québec" dans Recherches sociographiques, v. 36, n. 3, 1995, p. 479-526 (Revue publiée par le Département de sociologie de l'Université Laval à Québec ISSN 0034-1282).

CHRISTEN, Anne-Marie. "Le bilan des pratiques agroenvironnementales" dans L'Action Nationale, v. 86, n. 1, juin 1986, p. 63-84.

DEVERRE, Christian. "Social Implications of the agroenvironmental policy in France and Europe" dans la revue Sociologia Ruralis, v. 35, n. 2, 1995, p. 227-247.

ERVIN, D. E. "Some lessons about the political-economic effects of set-aside: the United States' experience" dans British Crop Protection Council, Monographs Series, v. 50, Farnham (Surrey), 1992, ISSN 0306-3941.

FAETH, Paul et al. Paying the Farm Bill: US Agricultural Policy and the Transition to Sustainable Agriculture, Washington, World Resources Institute, 1991.

GIRARD, Michel F. L'écologisme retrouvé. Essor et déclin de la Commission de la conservation du Canada, Ottawa, Les Presses de l'Université d'Ottawa, 1994, 308 p. (ISBN 2-7603-0379-9).

HILTS, Stewart, "Natural Heritage and Agricultural Production in Canada" dans I. R BOWLER, C. R. BRYANT; M. D. NELLIS. Contemporary rural systems in transition, Vol 1, Agriculture and Environnement, Wallingford (UK), C.A.B. International, 1992, p. 132-152.

JEAN, Bruno; Bernard VACHON. "Le développement rural et le modèle agricole productiviste" dans VACHON, Bernard (dir.), Le Québec rural dans tous ses états, Montréal, Éditions du Boréal, 1991, p. 149-166.

JEAN, Bruno. "Urbs contre rurs. L'agriculture durable ou la judiciarisation des pratiques agri-environnementales", dans le quotidien Le Devoir (Montréal), 9 décembre 1994, p. A-11 (en collaboration avec Andrée DESCHÊNES)
"La gestion des terres agricoles au Québec. Rationalité et irrationalité" dans France et Québec. Espaces ruraux en mutation, numéro thématique de Géographie sociale, v. 8, 1989, p. 71-80 (revue publié par le Centre de publications de l'Université de Caen).

"L'agriculture écologique" dans Agriculture et développement dans l'Est du Québec, Sillery, PUQ (Presses de l'Université du Québec), 1985, p. 319-350.

LAYCOCK, W. A. "The Conservation Reserve Program. How did we get where we are and where do we go from here?" dans L. A. JOYCE; J. E. MITCHELL; M. D. SKOLD (Eds.). The Conservation Reserve. Yesterday, Today ans Tomorrow, Fort Collins (Colorado), USDA Forest Service, Rocky Mountain Forest and range Experiment Station, 1991, p. 1-6.

MAPAQ (Ministère de l'Agriculture, des pêcheries et de l'alimentation du Québec). Le développement durable, pour la suite des choses. Politique ministérielle de développement durable, Québec, MAPAQ, 1995, 25 p.

NATIONAL RESEARCH COUNCIL - Committee on the Role of Alternative Farming Methods in Modern Production Agriculture. Alternative Agriculture, Washington DC, National Academy Press, 1989.

NELLIS, M. Duane; L. M. B. HARRINGTON; J. SHEELEY. "Policy, sustainability and scale: the U.S. Conservation Reserve Program", Texte de la communication présentée au Colloque de Charlotte (North Carolina), Groupe d'étude sur le développement durable des systèmes ruraux de l'Union géographique internationale en juillet 1995 .

NELLIS, Duane. "Agricultural Externalities and the Environment in the United States" dans I. R BOWLER; C.R. BRYANT; M. D. NELLIS. Contemporary rural systems in transition, Vol 1, Agriculture and Environnement, Wallingford (UK), C.A.B. International, 1992, p. 131-141.

OCDE. Politiques de l'agriculture et de l'environnement. Possibilités d'intégration, Paris, OCDE, 1989, 221 p.

L'intégration des politques de l'agriculture et de l'environnement: progrès récents et nouvelles orientations, Paris, OCDE, 1993, 114 p.

Pour une production agricole durable: des technologies plus propres, Paris, OCDE, 1994 (Coll. Documents OCDE), 103 p. 
Sylviculture, agriculture et environnement, Paris, OCDE, 1995, $208 \mathrm{p}$.

OSBORN, C. T.; R. KEIM. The Future Use of Conservation Reserve Program Acres: A National Survey of Farm Owners and Operators, Soil and Water Conservation Society, Ankeny, Indiana (USA), 1994.

PAUL, Morrie. La responsabilité en matière d'environnement dans le secteur agricole, Ottawa, Agriculture et Agroalimentaire Canada, Bureau de l'environnement, 1995, 58 p.

RIBAUDO, M..O.; S. PIPER et al. "CRP: What economic benefits?" dans Journal of Soil \& Water Conservation, v. 44 , n. 5 , p. $421-425$.
SOPUCK, Robert D. Politiques agricoles et commerciales du Canada: répercussions sur le reouveau rural et la biodiversité, Document de travail, Ottawa, Table ronde nationale sur l'Environnement et l'Économie (TRNEE), 1993, $61 \mathrm{p}$.

UNITED STATES OF AMERICA. Agricultural Reform and Improvement Act of 1996, Washington DC. Disponible sur le site Internet de la Library of Congress; voir http:// www.thomas.loc.gov/ (Connu sous le nom de Farm Bill). WICHEREK, Stanislas P.; Marc R. LAVERDIÈRE. "Les terres agricoles au Canada: dégradation et conservation" dans Cahiers Agricultures, v. 2, n.4, 1993, p. 245-255 (revue publié pour l'AUPEL-UREF par John Wiley Eurotext ISSN 1166-7699). 